\title{
RESTAURAÇÕES BIOLÓGICAS: UMA OPÇÃO DE TRATAMENTO PARA DENTES ANTERIORES FRATURADOS
}

\author{
BIOLOGICAL RESTORATIONS: AN \\ OPTION FOR FRACTURED ANTERIOR TEETH
}

\author{
Vanessa Cuman', Stella Kossatz Pereira² \\ 1 Autor para contato: Universidade Estadual de Ponta Grossa - UEPG, Campus em \\ Uvaranas, Departamento de Odontologia, Ponta Grossa, PR, Brasil; \\ e-mail: stellakp@ig.com.br; (42) 220-3111 e Universidade Paranaense - UNIPAR, \\ Umuarama, PR \\ 2 Praça Barão do Rio Branco, 259, apto 11 - Centro, Ponta Grossa, PR; \\ e-mail: vanessa.cuman@bol.com.br; fone: (42) 224-7110
}

Recebido para publicação em 16/04/2003 Aceito para publicação em 07/05/2003

\section{RESUMO}

Apesar da evolução dos materiais restauradores, não é possível restaurar com total fidelidade a estrutura dental perdida, quer seja por cárie ou por fratura, apenas com estes materiais. A possibilidade de reconstituição do elemento dental com um fragmento natural é um fator altamente positivo para o paciente, principalmente se considerarmos o fator emocional. Desta forma, o objetivo deste trabalho é apresentar uma alternativa às técnicas restauradoras convencionais, através da utilização do próprio elemento dental como material restaurador, ou seja, a técnica de colagem homógena. No caso clínico apresentado, o paciente teve a coroa do elemento dental 11 fraturada por trauma. Após tratamento endodôntico, foi cimentado um pino intracanal Fibrekor-Post (Jeneric/Pentron), para auxiliar na retenção do fragmento previamente preparado e autoclavado. A colagem foi realizada com o cimento resinoso dual Enforce (Dentsply). As avaliações clínica e radiográfica, realizadas após seis meses de acompanhamento, justificam o emprego dessa técnica.

Palavras-chave: fratura dental, colagem dentária, estética

\begin{abstract}
In spite of the evolution of restorative materials it is not possible to reconstitute with perfection the tooth lost because of caries lesions or coronary fractures. The possibility of the dental element to be rebuilt with a natural fragment is a highly positive factor because when fracture happens, the patient's and relative's emotional alterations are extremely strong. Thus the aim of this work is to present an alternative to the conventional restorative techniques, using the dental element itself as a
\end{abstract}


restorative material. The technique used was the reattachment of a tooth fragment which has a great fragment number of indications and advantages. In the clinical case presented in this study, the patient had fractured the dental crown number 11 as a result of a trauma. The endodontic treatment was performed according to the established techniques and an intra-channel pin was cemented using the FibrekorPost (Jeneric/Pentron). The homogeneous fragment was previously prepared. Right after that, the reattachment was performed using a dual cement Enforce (Dentsply). Six months later, clinical and radiographic evaluations were performed. The fragment remained in position and the aesthetic result obtained was favorable, facts that justify the use of this technique.

Keywords: dental fracture, dental bonding, aesthetics

\section{Introdução}

Proporcionar beleza ao sorriso é um dos principais objetivos da odontologia moderna. É crescente a exigência estética dos pacientes, sendo a sua satisfação um fator importante, pois denota o sucesso do tratamento. Com o passar do tempo, os conceitos estéticos têm sido aprimorados devido, principalmente, ao advento de novos materiais e técnicas restauradoras. Porém, apesar desta evolução, não é possível reconstituir com perfeição o dente perdido. Segundo Imparato et al. (1997), a introversão provocada por um sorriso desarmônico possui uma influência tão grande no ser humano, que altera a personalidade e pode prejudicar o comportamento social.

Chosack e Eidelman (1964), buscando uma alternativa para a recuperação estética de pacientes com dentes fraturados, propuseram a técnica de colagem de fragmentos com caráter provisório. Os autores recomendavam a manutenção dos fragmentos em solução salina para evitar a desidratação.

Esberard e Gabrielli (1978) estenderam a técnica proposta inicialmente como provisória para uma solução definitiva, associando-a aos materiais resinosos quimicamente ativados.

A técnica de colagem homógena foi descrita no Brasil por Gabrielli et al. (1981) que durante anos realizaram colagens de fragmentos através de um banco de dentes. Os fragmentos eram escolhidos de acordo com a cor, o tamanho e a forma. Os autores relataram que o aspecto imediatamente após as colagens revelava uma pequena diferença de cor entre o fragmento e o dente. Porém, esta desarmo- nia desaparecia entre 7 e 20 dias.

A principal indicação desta técnica é para dentes com fratura coronária ocasionada por trauma ou por lesão cariosa. Entre as vantagens, destaca-se o restabelecimento do elemento dental com suas características originais, manutenção da oclusão em dente natural, baixo custo e fator psicológico positivo, pois ocorre o resgate do bem estar emocional e social do paciente e de seus familiares.

Busato et al. (1984) apontaram como maior dificuldade desta técnica a seleção de dentes semelhantes ao remanescente coronário. Os fragmentos armazenados no banco de dentes devem ser hígidos, seccionados na porção coronária, estéreis e conservados em soro fisiológico (DUARTE et al., 1995; IMPARATO et. al., 1993; KRAMER et al., 1994; TAVARES et al., 1992).

No que diz respeito à equalização, alguns autores como Gabrielli et al. (1981); Gonzales e Busato (1994); Imparato et al. (1993); Santos et al. (1994) demonstraram que o fragmento recupera a cor, em média num período de 30 dias. Este processo depende, exclusivamente, das condições do paciente. $\mathrm{O}$ fato dos fragmentos apresentarem-se opacos inicialmente, deve-se à desidratação provocada fora do ambiente bucal (ESBERARD et al.; 1982; GABRIELLI et al., 1981; IMPARATO et al., 1993).

As restaurações biológicas representam um marco na ciência e na arte de restaurar dentes anteriores fraturados, pois permitem o aproveitamento do fragmento do próprio dente ou de um fragmento obtido a partir de um dente extraído (BARATIERI et al., 1995). 


\section{Relato de caso clínico}

Para a realização do caso clínico, obteve-se a autorização do Comitê de Ética da Universidade Estadual de Ponta Grossa - UEPG.

O paciente, A. D., 15 anos, apresentou-se à Clínica de Dentística Restauradora da Universidade Estadual de Ponta Grossa - UEPG, relatando fratura do elemento dental 11. Durante o exame clínico, verificou-se que o paciente apresentava boas condições periodontais, oclusão favorável e ausência de hábitos parafuncionais. Através do exame radiográfico, observou-se que o dente apresentava tratamento endodôntico satisfatório. Fatores essenciais à indicação da técnica.

Assim, na primeira sessão foi realizada profilaxia de todos os dentes com pedra-pomes e água em taça de borracha, seleção da cor do fragmento e da resina composta TPH Spectrum (Dentsply), regularização do remanescente dental e moldagem parcial da região ântero-superior com alginato Jeltrate (Dentsply). Através da moldagem, obtevese um modelo de gesso que auxiliou na escolha do fragmento dental, considerando-se tamanho e dimensão da coroa original.

A regularização do remanescente dental com ponta diamantada (KG 2135) foi realizada para facilitar a adaptação do fragmento à estrutura dental. O fragmento utilizado para a execução da técnica foi obtido através da doação de dentes pelas disciplinas de Cirurgia I e II, do Curso de Odontologia da Universidade Estadual de Ponta Grossa - UEPG. Os fragmentos empregados na técnica de colagem homógena são inicialmente imersos em Solução de Milton (hipoclorito de sódio 10\%) e seccionados 1 mm acima do limite amelodentinário com ponta diamantada (KG 2135). A câmara pulpar é esvaziada com ponta diamantada esférica (KG 1014) e o fragmento autoclavado a $134^{\circ} \mathrm{C}$ durante 30 minutos, seguindo o protocolo estabelecido pela disciplina de Dentística Restauradora da Universidade Estadual de Ponta Grossa - UEPG. Posteriormente, o dente é armazenado em geladeira dentro de um vidro tampado contendo soro fisiológico, que deve ser trocado semanalmente.
Na segunda sessão, nova profilaxia foi realizada nos dentes ântero-superiores, anestesia local, isolamento absoluto do campo operatório e preparo do canal radicular para cimentação do pino de fibra de vidro Fibrekor-Post (Jeneric/Pentron). Para tanto, a remoção inicial do material de obturação radicular foi realizada com a broca do sistema de pinos compatível ao diâmetro do canal. O desgaste das paredes internas do canal também foi realizado com a broca do sistema de pinos, considerando-se o diâmetro do pino selecionado. Em seguida, o condicionamento ácido do remanescente dental e do fragmento preparado foi realizado com ácido fosfórico 37\% durante 15 segundos. Após a remoção com jato de água e secagem com ar das estruturas condicionadas, aplicou-se o sistema adesivo Prime \& Bond 2.1 (Dentsply). Vale ressaltar que no interior do canal radicular, o mesmo sistema adesivo foi aplicado em associação ao Self Cure Activator (Dentsply), para garantir a polimerização química do mesmo. O pino de fibra de vidro foi silanizado com Silano Primer e Ativador (Dentsply), seguindo as recomendações do fabricante. A cimentação do pino foi realizada com o cimento resinoso dual Enforce (Dentsply).

O tratamento dado ao fragmento dental - após o condicionamento ácido - foi semelhante ao protocolo realizado no remanescente dental e sua cimentação foi realizada com o mesmo cimento resinoso dual Enforce (Dentsply) empregado para a cimentação do pino. Com o propósito de preencher os espaços na linha de cimentação entre remanescente dental e fragmento utilizou-se a resina composta TPH Spectrum (Dentsply) em incrementos de $1 \mathrm{~mm}$ fotopolimerizados durante 40 segundos com o aparelho Ultralux (Dabi Atlante) apresentando intensidade de luz média de $400 \mathrm{~mW} / \mathrm{cm}^{2}$. $\mathrm{O}$ ajuste oclusal foi aferido com filme carbono Detecto (Dentsply).

O polimento final da resina composta foi realizado 48 horas após a colagem do fragmento utilizando-se pontas de silicone Enhance (Dentsply) e discos de lixa Sof-Lex (3M) montados em baixa rotação. Toda a seqüência clínica está ilustrada nas Figuras 1 a 12. 


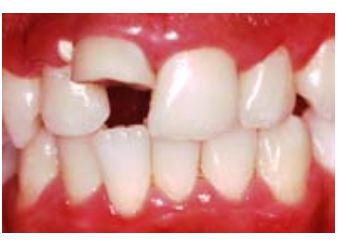

Figura 1 - Visão clínica inicial

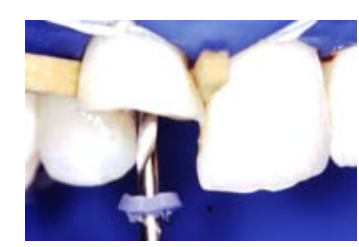

Figura 4 - Utilização da broca média do kit Fibrekor-Post (Jeneric/Pentron)

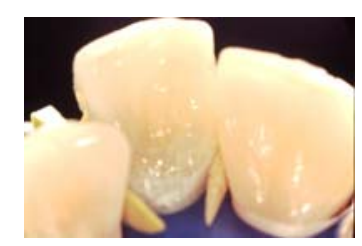

Figura 7 - Vista palatina após colagem

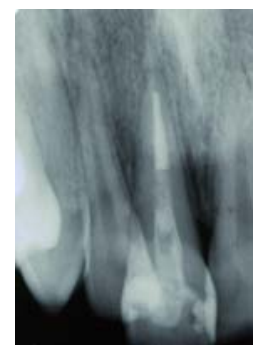

Figura 10 - Radiografia final

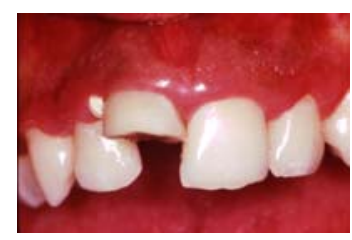

Figura 2 - Vista aproximada

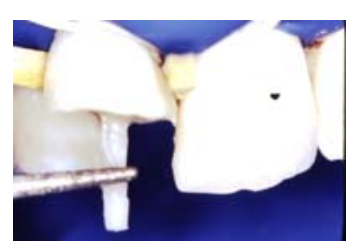

Figura 5 - Corte do pino Fibrekor-Post (Jeneric/Pentron) com a ponta KG (2035)

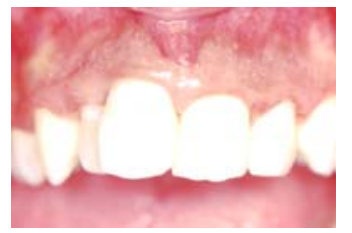

Figura 8 - Aspecto final após polimento

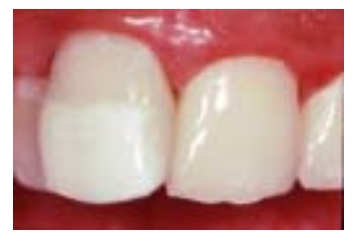

Figura 11 - Aspecto após 6 meses

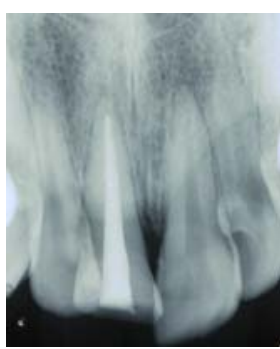

Figura 3 - Radiografia inicial

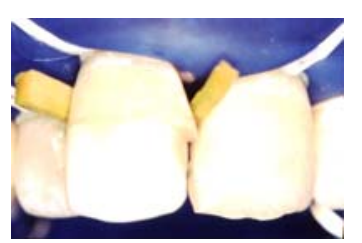

Figura 6 - Utilização do cimento resinoso dual Enforce (Dentsply)

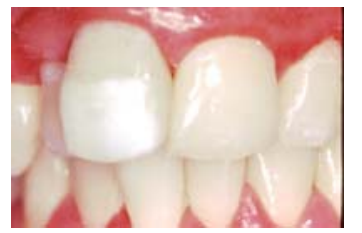

Figura 9 - Vista aproximada após polimento

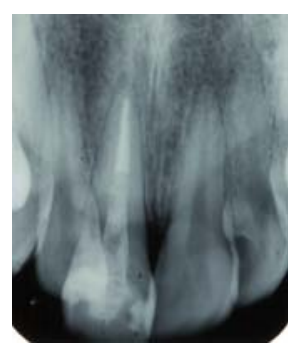

Figura 12 - Radiografia após 6 meses 


\section{Resultados}

O resultado obtido imediatamente após a colagem revelou estética favorável com pequena diferença de cor entre o remanescente e o fragmento dental. Com relação à equalização observou-se que 15 dias após à colagem, a cor do fragmento estava bastante próxima da cor do remanescente dental.

Após 6 meses, avaliações clínica e radiográfica foram executadas onde se constatou que a estética continuava favorável, não tendo ocorrido deslocamento do fragmento ou manchamento da resina composta. Entretanto, pôde-se observar uma pequena mancha de coloração branco leitosa na interface fragmento/remanescente dental, sugerindo o extravasamento do cimento resinoso nesta área.

Neste período de acompanhamento, não se detectou comprometimento na retenção do fragmento através do pino intracanal, cimento resinoso, resina composta ou sistema adesivo, o que indicou o sucesso da técnica proposta. O resultado obtido foi excelente, justificando o procedimento e concordando com os relatos de Gabrielli et al. (1981); GONZALES et al. (1994); IMPARATO et al. (1993); KOZEN et al. (1990).

A diferença de cor foi reduzida após 15 dias através da hidratação do fragmento pela saliva, concordando com os estudos de Gonzales et al. (1994); Santos et al. (1994).

A maior dificuldade para o emprego desta técnica é a seleção de dentes para a colagem. O fragmento escolhido deve ser semelhante aos outros dentes do paciente e ter dimensões que possibilitem um ajuste satisfatório entre este e o remanescente dental (BUSATO et al. 1984; KRAMER et al. 1994).

\section{Conclusão}

Os resultados obtidos após 6 meses de avaliações clínica e radiográfica nos permitem concluir que a técnica de restauração biológica representa uma opção satisfatória para o restabelecimento estético e funcional de dentes anteriores, desde que seja corretamente indicada e executada de acordo com o protocolo clínico estabelecido.

\section{Agradecimentos}

Às empresas Dentsply e Jeneric/Pentron, pelos materiais utilizados neste trabalho.

\section{REFERÊNCIAS}

1 BARATIERI, L. N.; MONTEIRO JUNIOR, S.;ANDRADA, M. A. C.; VIEIRA, L. C. C.; CARDOSO, A. C.; RITTER, A. V. Estética: Restaurações adesivas diretas em dentes anteriores fraturados. São Paulo: Santos, 1995. p.137-205.

2 BUSATO, A. L. S. e ANTUNES, M. Colagem homógena em dentes anteriores fraturados. RGO - Revista gaúcha de odontologia, v. 32, p. 137 - 140, 1984.

3 CHOSACK, A. e EIDELMAN, E. Rehabilitacion of fractured incisal resing the patients natural crown. Case report. J. Dent. Child, v. 31, p. 19-21, 1964.

4 DUARTE, D. A.; IMPARATO, J. C. P.; GUEDES PINTO, A. C. Colagem de fragmento dentário em molares decíduos. APCD - Revista da associação paulista de cirurgiõesdentistas, v. 49, n. 1, p. 76-78, 1995.

5 ESBERARD, R. M. e SILVA FILHO, F. P. Caso Clínico: fratura coronária em dente anterior. APCD - Revista da associação paulista de cirurgiões-dentistas, v. 32, p. 12033, 1978.

6 ESBERARD, R. M. e SILVA FILHO, F. P. Restaurações de dentes anteriores fraturados com aproveitamento de fragmento. RGO - Revista gaúcha de odontologia, v. 30, p. 9-103, 1982.

7 GABRIELLI, F.; DINELLI, W.; FONTANA, U. F.; PORTO, C. L. A. Apresentação e avaliação clínica de uma técnica de restauração, de dentes anteriores, com fragmento adaptados de dentes extraídos. RGO - Revista gaúcha de odontologia, v. 29, n. 2, p. 83-87, 1981.

8 GONZALES, P. A. H. e BUSATO, A. L. S. Restaurações biológicas com fragmentos dentários. Revista brasileira de odontologia, v. 51, n. 2, 1994.

9 IMPARATO, J. C. P.; PAIXÃO, R. F.; DUARTE, D.A.; GUEDES PINTO, A.C. Restaurações atípicas em molares decíduos através de colagem de fragmentos dentários. Revista de odontopediatria - atualização e clínica, v. 4, n. 2, p. 219$225,1993$. 
10 IMPARATO, J. C. P.; DUARTE, D. A.; COMISSOLI, L.; BUSSADORI, S. K. Faceta estética de esmalte em dentes decíduos anteriores. Jornal brasileiro de odontologia clínica, v. 1, p. 55-59, 1997.

11 KOZEN, V. e BUSATO, A. L. S. Coroa total com dente natural. RGO - Revista gaúcha de odontologia, v. 38, n. 3, p. 195-206, 1990.

12 KRAMER, P. F. e UNIKOVSKI, C. P. Colagem heterógena em dentes decíduos posteriores. Revista de odontopediatria
- atualização e clínica, v. 3, n. 1, p. 35-41, 1994.

13 SANTOS, J. F. F. e BIANCHI, J. Restauration of several damaged teeth whith resin bonding system - case reports. Quintessence International, v. 22, n. 8, p. 611-615, 1994.

14 TAVARES, A. C.; GOES, W. A.; PAIXÃO, R. F.; IMPARATO, J. C. P. Reconstrução de dente decíduo posterior utilizando fragmento dentário humano. Relato de um caso clínico. Revista da faculdade de odontologia de Fortaleza, v. 4, n. 2, p. 113-117, 1992. 